\title{
Substitutional Si Doping of Graphene and Nanotubes through Ion Irradiation- Induced Vacancies
}

\author{
Heena Inani $^{1 *}$, Kimmo Mustonen ${ }^{1}$, Alexander Markevich ${ }^{1}$, Er-Xiong Ding ${ }^{2}$, Mukesh Tripathi ${ }^{1}$, Aqeel \\ Hussian$^{2}$, Clemens Mangler, Esko I. Kauppinen ${ }^{2}$, Toma Susi ${ }^{1}$ and Jani Kotakoski ${ }^{1}$ \\ 1. University of Vienna, Faculty of Physics \& VDSP, Vienna, Austria. \\ 2. Aalto School of Science, Department of Applied Physics, Aalto, Finland. \\ * Corresponding Author: heena.inani@univie.ac.at
}

Low-dimensional materials have unique intrinsic properties as a result of their electronic wave-function confinement in one or two dimensions. For example, the electronic properties of single-walled carbon nanotubes (SWCNTs) entirely depend on the rolling direction of the graphene sheet. Unfortunately, the synthesis of nanotubes with selective chirality remains a challenge despite enormous efforts during the past decades. Another way to obtain specific properties is to introduce heteroatom dopants into the lattice [1]. Although covalently bound nitrogen (N) [2], boron (B) [2], silicon (Si) [3] and germanium (Ge) [4] have been demonstrated in graphene, direct evidence for SWCNTs is for the most part missing, except for $\mathrm{N}$ substitution [5].

We present here atomic-scale evidence of the substitution of Si heteroatoms in graphene and carbon nanotubes through Ar plasma irradiation assisted with simultaneous laser irradiation. Scanning transmission electron microscopy (STEM) imaging and electron energy loss spectroscopy allow us to directly identify the heteroatoms, their bonding environment and configuration in the materials. A graphene-SWCNTs heterostructure was used as a target material, with nanotubes synthesized using a floating catalyst method and dry-deposited on graphene [6]. The samples were introduced into the vacuum system connected to the Nion UltraSTEM 100 microscope in Vienna [7], and characterized at atomic resolution. Due to typical hydrocarbon contamination, the samples were cleaned with laser [8] ( $6 \mathrm{~W}, 445 \mathrm{~nm}$ power tunable diode laser) in the microscope column at a pressure of $\sim 10^{-10}$ mbar. Clean areas of pristine graphene and SWCNTs are shown in Fig. 1a and Fig. 2a, respectively. The samples were next transferred (in vacuum) to a manipulation setup, where Ar plasma was ignited in a microwave cavity and accelerated to a total kinetic energy of $\sim 50 \mathrm{eV}$ towards the sample with simultaneous highpower laser irradiation.

Ar ion impacts lead to the production of defects in both graphene and carbon nanotubes. At ion energies used here, mostly single and double vacancies are expected [9]. The simultaneous laser heating keeps the samples clean and provides thermal energy for $\mathrm{C}$ and $\mathrm{Si}$ atoms of the remaining contamination to be released and diffuse on the sample. After plasma treatment, atomic-scale analysis reveals that indeed a large number of Si atoms has entered both structures. We found both 3- and 4-coordinated Si atoms in SWCNTs [10] as shown in Fig. 2(b-c) as well as in graphene [3]. Fig. 1b shows plasma-irradiated graphene with substituted Si atoms. The concentration of Si atoms in graphene and SWCNTs was found to be $0.3 \%$ and $0.1 \%$, respectively [10]. The recurrence of 3- and 4-coordinated configurations was found to be $69 \%$ and $37 \%$ in SWCNTs [10], respectively, in a good agreement with the predicted ratio of single and double vacancies created by $50 \mathrm{eV}$ Ar irradiation $[9,11]$.

References: 
[1] O Stephan et al., Science 266 (1994), p. 1683.

[2] U Bangert et al., Nano Lett. 13 (2013), p. 4902.

[3] QM Ramasse et al., Nano Lett. 13 (2013), p. 4989.

[4] M Tripathi et al., ACS Nano 12 (2018), p. 4641.

[5] R Arenal et al. Nano Lett. 14 (2014), p. 5509.

[6] K Mustonen et al. ACS Nano 12 (2018), p. 8512.

[7] GT Leuthner et al., DOI: j.ultramic.2019.02.002 (2019).

[8] M Tripathi et al., Physica Status Solidi RRL 11 (2017), p. 1700124.

[9] A Tolvanen et al., Appl. Phys. Lett. 91 (2007), p. 173109.

[10] H Inani et al., arXiv:1902.02611

[11] The authors acknowledge funding by the FWF project I3181-N36, P 28322-N36 and P31605, Wiener Wissenschafts- Forschungs- und Technologiefonds (WWTF) project MA14-009, European Research Council (ERC) Grant No. 756277-ATMEN, Finnish Cultural Foundation through a grant from the Finnish Postdoc Pool and projects 286546DEMEC, 292600-SUPER, 3303/31/2015 (CNT-PV), 1882/31/2016 (FEDOC) and the Aalto Energy Efficiency (AEF) Research Program through the MOPPI project. Ms. Heena Inani is thankful to Vienna Doctoral of Physics for providing additional support.

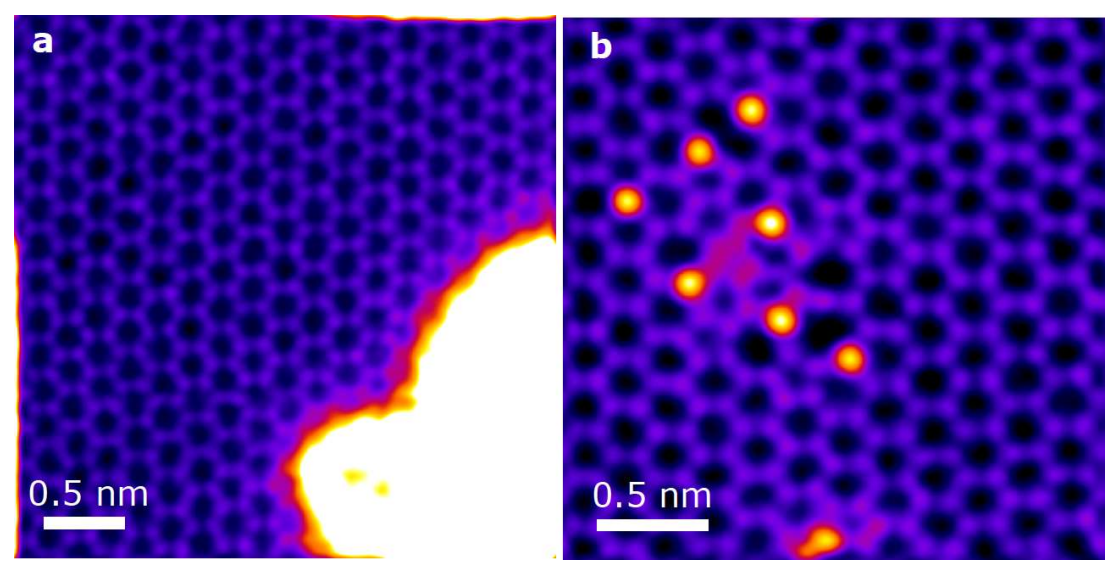

Figure 1. Atomically resolved images (STEM medium angle annular dark field (MAADF)) of (a) pristine graphene and (b) plasma-irradiated graphene (bright atoms are $\mathrm{Si}$ ).
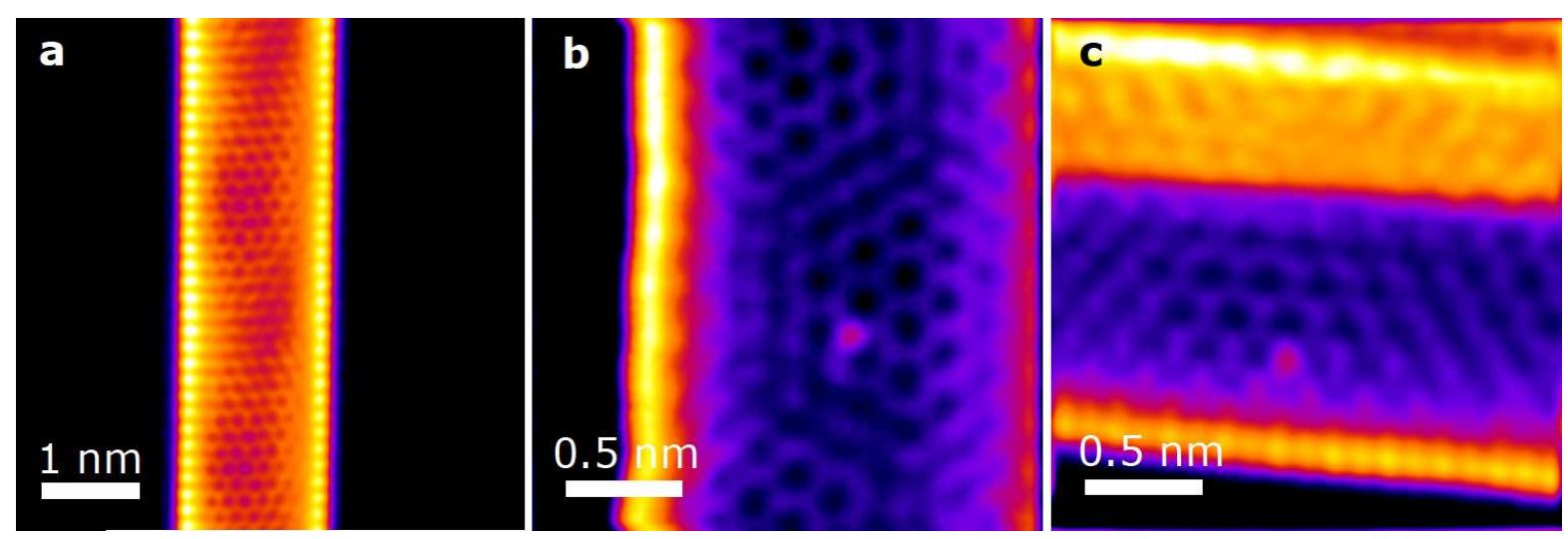

Figure 2. STEM/MAADF images of (a) a pristine SWCNT, (b) 3-coordinated Si atom and (c) 4coordinated Si atom in plasma-irradiated SWCNTs. 